\title{
Plasma profile and urine excretion of amino acids in children with celiac disease on gluten-free diet after oligofructose-enriched inulin intervention: results of a randomised placebo-controlled pilot study
}

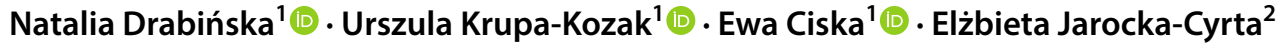

Received: 9 April 2018 / Accepted: 16 July 2018 / Published online: 24 July 2018

(c) The Author(s) 2018

\begin{abstract}
The circulating amino acid (AAs) concentrations are indicators of dietary protein intake and metabolic status. In celiac disease (CD), the AA imbalance is frequently observed. Prebiotics are found to alleviate nutrient deficiencies. Therefore, the aim of this study was to analyse the impact of oligrofructose-enriched inulin (Synergy 1), administered for 3 months as a gluten-free diet (GFD) supplement to children with CD, on the plasma and urine concentrations of AAs. CD children $(N=34)$ were randomised into two groups, receiving Synergy 1 ( $10 \mathrm{~g} /$ day) or placebo (maltodextrin) for 3 months. The AA profile and concentration was determined in plasma and urine before and after the dietary intervention by gas chromatography. 22 and 28 AAs were determined in plasma and urine samples, respectively. After the intervention, the plasma concentrations of several AAs (Ala, Pro, Asn, Glu, Tyr, Lys, His, Orn) increased significantly in both experimental groups, while Gln increased only in the Synergy 1 group. The urinary excretion of Asn, Lys and Aaa increased significantly in the Synergy 1 group, and the excretion of Asp and Met decreased $(p<0.05)$ in the placebo group. The Gln:Glu ratio in urine increased in both groups after the intervention. An increased urinary excretion of AAs observed in Synergy 1 group with a simultaneous increase in the content of circulating AAs could be attributed to higher absorption or intensified metabolism of AAs, and on the other hand further healing of the intestinal mucosa being the result of continuous treatment with GFD. Moreover, the observed changes in Glu concentration suggest that oligofructose-enriched inulin could improve the intestinal condition and permeability. To conclude, a prebiotic-supplemented GFD influences beneficially the overall AAs metabolism in CD children; however, further prospective cohort studies are needed to confirm the results obtained.
\end{abstract}

Keywords Amino acids $\cdot$ Celiac disease $\cdot$ Oligofructose-enriched inulin $\cdot$ Dietary intervention $\cdot$ Clinical trial $\cdot$ Gluten-free diet

\section{Introduction}

Chronic intestinal inflammation alters gut functions by weakening the intestinal barrier, damaging epithelial cells and decreasing absorption (Barker and Liu 2008), which

Handling Editor: F. Blachier.

Natalia Drabińska

n.drabinska@pan.olsztyn.pl; natalia_drabinska@wp.pl

Urszula Krupa-Kozak

u.krupa-kozak@pan.olsztyn.pl

Ewa Ciska

e.ciska@pan.olsztyn.pl

Elżbieta Jarocka-Cyrta

ejarocka@op.pl

1 Department of Chemistry and Biodynamics of Food, Institute of Animal Reproduction and Food Research of Polish Academy of Sciences, Tuwima 10 Str., 10-748 Olsztyn, Poland

2 Department of Pediatrics, Gastroenterology and Nutrition, Collegium Medicum, University of Warmia \& Mazury, Oczapowskiego 2, Str., 10-719 Olsztyn, Poland 
deplete the levels of important nutrients and mediators in the human body, including amino acids (AAs). AAs are essential metabolites which play a crucial role in the human body. They participate in the formation of protein structure, regulation of anabolic and catabolic metabolism, and detoxification processes. Dietary AAs act as substrates for the development of epithelial cells and mucin synthesis, they maintain the integrity of the gut barrier and play a pivotal role in the intestinal immune system (Fillmann et al. 2007; Ren et al. 2014; Zhang et al. 2015). AAs are the precursors for the synthesis of important mediators, such as glutathione, an antioxidant with cytoprotective activity which prevents epithelial cell damage (Wu et al. 2004). The concentrations of circulating AAs are reliable indicators of dietary protein intake and metabolic status. Recent research has focused on the AA balance and changes in AA metabolism as factors that contribute to the development of pathological conditions. Changes in the AA profile often accompany cancerogenesis, mental disorders and fatigue (Evans et al. 2008; Hugh Dunstan et al. 2011; Dereziński et al. 2017). The AA imbalance is also frequently observed in celiac disease (CD) (Di Cagno et al. 2011; Sevinc et al. 2015).

$\mathrm{CD}$ is a chronic autoimmune enteropathy which is triggered by gluten ingestion and is observed in genetically predisposed individuals. The only treatment for $\mathrm{CD}$ is a gluten-free diet (GFD) which may be difficult to optimise in terms of fulfilling the patients' nutritional needs (Roma et al. 2010; Ilus et al. 2014). CD patients who have been following a GFD for a long time are characterised by suboptimal levels of essential AAs (EAAs) in biological fluids, compared with healthy individuals (van Hees et al. 2015). The psychological symptoms that accompany $\mathrm{CD}$, such as anxiety, irritability, depression and apathy (Carta et al. 2002; Addolorato et al. 2008), are linked with a reduced intake of dietary tryptophan and, consequently, decreased synthesis of monoamines, including serotonin (Hernanz and Polanco 1991). According to many metabolic studies (Bertini et al. 2009; Di Cagno et al. 2009), the AA balance plays a very important role in CD management. Despite the above, AA profiling in $\mathrm{CD}$ has been scarcely researched (Sevinc et al. 2015).

According to the literature, prebiotics could be helpful in alleviating nutrient deficiencies by regulating the composition and the activity of gut microbiota. In vitro and in vivo studies as well as clinical trials involving patients with intestinal inflammatory diseases have demonstrated that inulin-type fructans improve mineral absorption, alleviate the symptoms of inflammation and induce morphological changes in the intestines (Guarner 2007; KrupaKozak et al. 2016; Shoaib et al. 2016). The applicability of inulin-type fructans in GFD and its effects on the AA profile in biological fluids have not been investigated to date. Therefore, the aim of this study was to analyse the impact of oligrofructose-enriched inulin, administered for 3 months as a GFD supplement to children with $\mathrm{CD}$, on the plasma and urine concentrations of selected AAs.

\section{Materials and methods}

\section{Experimental design}

A randomised, placebo-controlled, parallel-group and single-centre pilot study was performed on children and adolescents $(N=34$, aged from 4 to 18 years) diagnosed with $C D$ based on the criteria of the European Society for Paediatric Gastroenterology, Hepatology and Nutrition (ESPGHAN) (Husby et al. 2012), and following GFD since at least 6 months (GFD adherence in a range from 6 months to 9 years) to assess the effects of oligofructose-enriched inulin (Synergy 1; Orafti ${ }^{\circledR}$ Synergy 1, Beneo, Tienen, Belgium) on their health and nutritional status. All participants met the inclusion criteria: $\mathrm{CD}$ confirmed by serological, genetic and biopsy analyses; GFD for at least 6 months. The exclusion criteria included: use of antibiotics in the month preceding the study; use of medication for osteoporosis (bisphosphonates, calcium calcitonin); use of probiotics, prebiotics or fibre supplements; poor or average overall health; current enrolment in another research study; recent surgery. The detailed protocol of the clinical trial, and the demographic and anthropometric characteristics of the participants at the beginning of the study have been described previously (Krupa-Kozak et al. 2017). The patients were randomised into two groups: based on sex and age: the Synergy 1 group $(N=18$; girls $=11$; age $5-17$ years, average 10 years $)$ and the placebo group $(N=16$; girls $=10$; age $4-16$ years, average 10 years). Over a period of 3 months, the participants in the Synergy 1 group were administered oligofructose-enriched inulin at $10 \mathrm{~g} / \mathrm{day}$, and the participants in the placebo group were administered maltodextrin at $7 \mathrm{~g} /$ day. Patients of both groups continued to follow a strict GFD throughout the whole experiment. Among recruited subjects, 30 completed the study and were included in the final analysis, while four children were excluded, in particular two subjects who had been treated with antibiotics during the experiment and two subjects who failed to comply with the experimental requirements.

Anti-tissue transglutaminase antibody (tTG) assay has been performed before and after the intervention as a part of clinical practice by Hospital's Diagnostic Laboratory to control adherence to GFD and/or progress of the treatment. The results of tTG assay are presented in Table 1. 
Table 1 Values of anti-tissue transglutaminase in CD children before and after the intervention, expressed as a median (P25-P75). Normal range is $<8.0 \mathrm{AU} / \mathrm{mL}$

\begin{tabular}{llllll}
\hline & T0 & & & T1 & \\
\cline { 2 - 3 } \cline { 5 - 6 } & Synergy 1 & & Slacebo & Synergy 1 & Placebo \\
\hline tTG $[\mathrm{AU} / \mathrm{mL}]$ & $2.57(0.48-6.98)$ & $2.17(0.85-3.74)$ & & $1.22(0.34-4.88)$ & $1.49(0.62-4.83)$ \\
\hline
\end{tabular}

\section{Sample collection}

Urine and blood samples were collected from each participant at baseline and after the 3-month dietary intervention. For ethical reasons, samples were collected during regular follow-up visits (every 3 months) in the Gastroenterology Clinic of the Children's Hospital in Olsztyn (Poland). Blood samples were collected into vacuum tubes containing an anticoagulant (heparin). Plasma was divided into aliquots $(100 \mu \mathrm{L})$ and stored at $-80{ }^{\circ} \mathrm{C}$ until AA analyses. Approximately $50 \mathrm{~mL}$ of morning urine (second spontaneous urine) was collected, divided into aliquots and stored at $-80{ }^{\circ} \mathrm{C}$ until further analysis.

\section{Amino acid analysis}

The AA profile in urine and plasma samples was analysed using the EZ:Faast ${ }^{\mathrm{TM}}$ Kit for Free (Physiological) Amino Acids (Phenomenex, Aschaffenburg, Germany) according to the producer's recommendations. The chromatographic method was chosen due to its ability to detect a wide range of AAs in a single run and in a very short time (7 min) using a relatively small amount of the sample. The analytical procedure involves solid-phase extraction of $100 \mu \mathrm{L}$ of urine or plasma, followed by derivatization and liquid-liquid extraction. AAs were identified in the Agilent 7890A gas chromatograph (Agilent Technologies, Santa Clara, CA, USA) coupled with the 5975C mass selective detector, 7683B auto-injector and a data station containing the NIST/EPA/NIH Mass Spectral Library (Version 2). The compounds were separated in the ZBAAA EZ Faast ${ }^{\mathrm{TM}}$ capillary column $(10 \mathrm{~m} \times 0.25 \mathrm{~mm})$. The carrier gas was helium $(1.5 \mathrm{~mL} / \mathrm{min})$. The samples $(2 \mu \mathrm{L})$ were injected in split mode $(1: 15)$. Oven temperature was initially set at $110{ }^{\circ} \mathrm{C}$ and then increased to $320{ }^{\circ} \mathrm{C}$ $\left(30{ }^{\circ} \mathrm{C} / \mathrm{min}\right)$. Injector and MS source temperatures were 250 and $240{ }^{\circ} \mathrm{C}$, respectively. Mass spectra were obtained by electron ionisation (EI) over the range of $35-550 \mathrm{~m} / \mathrm{e}$. Electronic impact energy was $70 \mathrm{eV}$. Amino acids were identified using calibration standards for each AA, and a quantitative analysis was performed relative to the internal standard (norvaline).

\section{Statistical analysis}

All analyses were performed in duplicates. Raw data were expressed as means \pm SEM. The Gln:Glu ratio was presented as means \pm SD. Data were processed in Statistica 12 software (StatSoft, USA). Only the data collected from participants whose reported intake of the supplements exceeded $80 \%$ were included in the final analysis. Normal distribution was evaluated in the Shapiro-Wilk test. Analyses comparing both groups at baseline and after 3 months were performed separately using the Student's $T$ test or the Mann-Whitney $U$ test as appropriate. Comparison within groups between baseline and after 3 months was performed using the paired samples Student's $T$ test or Wilcoxon test, as appropriate. Statistical significance thresholds were set at $p<0.05\left(^{*}\right)$ and $p<0.01\left(^{* *}\right)$.

\section{Results}

\section{Amino acid profile in plasma}

Twenty-two AAs, including eight non-essential AAs (NEAAs) (Ala, Gly, Ser, Pro, Asn, Gln, Glu, Tyr), nine EAAs (Val, Leu, Ile, Thr, Met, Phe, Lys, Trp, His) and five non-proteinogenic AAs and derivatives (Aba, Orn, Hyp, $\mathrm{C}-\mathrm{C}, \mathrm{Php}$ ), were determined in plasma samples collected from children with $\mathrm{CD}$ (Table 2). At baseline, the plasma profile and the concentrations of individual AAs and total AA in did not differ between the Synergy 1 group and the placebo group.

After the 3-month dietary intervention, the concentrations of NEAAs, in particular Ala, Pro, Asn, Glu, Tyr, as well as Lys, His and Orn increased significantly $(p<0.05)$ in both groups, regardless of the applied GFD supplementation (Table 2). The concentration of Gln increased significantly $(p<0.05)$ only in Synergy 1 group. On the other hand, a significant increase of the concentration of majority of EAAs and of Aba, Hyp and C-C in plasma was observed only in the placebo group. Interestingly, the administration of Synergy 1 significantly increased $(p<0.01)$ the plasma Glu level, but did not affect the Gln:Glu ratio which remained similar to baseline (Fig. 1a). Consequently, the total AA 
Table 2 Concentrations of AAs and derivatives in the plasma of children with celiac disease $(N=30)$ : a comparison of the group supplemented with Synergy 1 and the placebo group at baseline and after 3 months of supplementation

\begin{tabular}{|c|c|c|c|c|c|c|c|c|c|c|c|c|}
\hline & & \multicolumn{4}{|l|}{ T0 } & \multicolumn{4}{|l|}{$\mathrm{T} 1$} & \multirow[t]{3}{*}{ S-T0 vs. S-T1 } & \multirow[t]{3}{*}{ P-T0 vs. P-T1 } & \multirow[t]{3}{*}{ S-T1 vs. P-T1 } \\
\hline & & \multicolumn{2}{|c|}{ Synergy 1 (S) } & \multicolumn{2}{|c|}{ Placebo (P) } & \multicolumn{2}{|l|}{$\mathrm{S}$} & \multicolumn{2}{|l|}{$\mathrm{P}$} & & & \\
\hline & & $\mathrm{Av}$ & SEM & $\mathrm{Av}$ & SEM & $\mathrm{Av}$ & SEM & $\mathrm{Av}$ & SEM & & & \\
\hline \multicolumn{13}{|l|}{ Non-essential amino acids } \\
\hline Alanine & Ala & 284.70 & 21.73 & 280.96 & 20.76 & 387.94 & 22.48 & 406.25 & 30.91 & $0.008 * *$ & $0.012^{*}$ & 0.629 \\
\hline Glycine & Gly & 226.80 & 20.58 & 210.69 & 16.33 & 248.97 & 16.50 & 229.08 & 16.97 & 0.110 & 0.286 & 0.325 \\
\hline Serine & Ser & 109.19 & 7.72 & 93.40 & 5.65 & 123.91 & 10.26 & 119.36 & 8.65 & 0.196 & $0.008 * *$ & 1.000 \\
\hline Proline & Pro & 137.27 & 6.48 & 129.46 & 9.59 & 164.65 & 9.57 & 199.39 & 19.38 & $0.028 *$ & $0.007 * *$ & 0.197 \\
\hline Asparagine & Asn & 46.89 & 3.28 & 43.62 & 3.30 & 54.52 & 2.70 & 57.89 & 2.72 & $0.006 * *$ & $0.008^{*}$ & 0.395 \\
\hline Glutamine & Gln & 457.39 & 25.48 & 411.34 & 18.35 & 498.93 & 16.11 & 473.37 & 36.35 & $0.015^{*}$ & 0.092 & 0.485 \\
\hline Glutamic acid & Glu & 22.86 & 2.65 & 25.69 & 2.85 & 48.63 & 2.43 & 39.41 & 2.86 & $0.007 * *$ & $0.043^{*}$ & $0.037 *$ \\
\hline Tyrosine & Tyr & 43.67 & 2.64 & 41.01 & 2.40 & 54.19 & 3.84 & 61.14 & 6.06 & $0.030^{*}$ & $0.016^{*}$ & 0.433 \\
\hline \multicolumn{13}{|l|}{ Essential amino acids } \\
\hline Valine & Val & 225.65 & 18.04 & 202.43 & 15.13 & 241.97 & 11.91 & 244.09 & 24.88 & 0.743 & 0.145 & 0.936 \\
\hline Leucine & Leu & 101.49 & 7.79 & 85.91 & 4.01 & 110.78 & 8.87 & 124.23 & 15.55 & 0.182 & $0.008 * *$ & 0.761 \\
\hline Isoleucine & Ile & 51.36 & 3.33 & 45.51 & 2.33 & 53.29 & 1.98 & 57.34 & 4.50 & 0.541 & 0.084 & 0.363 \\
\hline Threonine & Thr & 110.59 & 7.94 & 105.11 & 11.01 & 136.35 & 9.31 & 137.46 & 9.78 & 0.112 & $0.047 *$ & 0.935 \\
\hline Methionine & Met & 11.53 & 0.88 & 10.02 & 0.66 & 13.52 & 0.65 & 14.65 & 1.72 & 0.071 & $0.012 *$ & 0.483 \\
\hline Phenylalanine & Phe & 35.81 & 1.70 & 35.35 & 1.64 & 39.15 & 2.50 & 41.20 & 1.98 & 0.186 & $0.034 *$ & 0.542 \\
\hline Lysine & Lys & 193.74 & 8.27 & 182.73 & 6.81 & 247.26 & 10.06 & 267.94 & 14.36 & $0.011^{*}$ & $0.007 * *$ & 0.240 \\
\hline Tryptophan & $\operatorname{Trp}$ & 43.23 & 2.49 & 39.21 & 1.89 & 46.30 & 3.46 & 55.39 & 4.43 & 0.051 & $0.018 *$ & 0.070 \\
\hline Histidine & His & 65.28 & 3.12 & 61.36 & 3.65 & 75.16 & 3.11 & 86.36 & 6.72 & $0.042 *$ & $0.016^{*}$ & 0.218 \\
\hline \multicolumn{13}{|c|}{ Other amino acids and derivatives } \\
\hline$\alpha$-Aminobutyric acid & Aba & 17.07 & 1.27 & 15.43 & 1.11 & 17.88 & 1.44 & 19.36 & 1.75 & 0.421 & $0.011^{*}$ & 0.685 \\
\hline Ornithine & Orn & 47.85 & 2.14 & 42.45 & 2.79 & 59.71 & 2.48 & 72.95 & 7.69 & $0.012^{*}$ & $0.002 *$ & 0.174 \\
\hline 4-hydroxyproline & Hyp & 20.03 & 2.78 & 18.79 & 2.22 & 22.07 & 1.88 & 27.14 & 3.04 & 0.955 & $0.008 * *$ & 0.164 \\
\hline Cystine & $\mathrm{C}-\mathrm{C}$ & 25.94 & 3.81 & 23.89 & 3.34 & 25.08 & 3.22 & 35.39 & 4.31 & 0.953 & $0.028 *$ & 0.193 \\
\hline Proline-hydroxyproline & Php & 9.44 & 1.27 & 6.89 & 1.30 & 8.42 & 2.64 & 10.82 & 3.22 & 0.463 & 0.089 & 1.000 \\
\hline
\end{tabular}

Data are given as the mean with SEM in nmol/mL. Differences were considered significant as follow: $(*) p<0.05 ;(* *) p<0.01$

concentration increased by $17 \%$ in the Synergy 1 group and by $32 \%$ in the placebo group relative to baseline (Fig. 2a). The total concentration of EAAs increased by $15 \%$ in the Synergy 1 group and by $23 \%$ in the placebo group, and the total concentration of NEAAs increased by $19 \%$ in both groups. The total concentration of branched-chain amino acids (BCAAs) increased by $7 \%$ in the Synergy 1 group and by $13 \%$ in the placebo group.

\section{Amino acid profile in urine}

The urinary AA profile determined in Synergy 1 and placebo groups at baseline and after the dietary intervention is presented in Table 3. Twenty-eight AAs, including nine NEAAs (Ala, Gly, Ser, Pro, Asn, Asp, Gln, Glu, Tyr), nine EAAs (Val, Leu, Ile, Thr, Met, Phe, Lys, Trp, His) and ten AA derivatives and non-proteinogenic AAs (Aba, $\beta$ AiB, Aaa, Apa, Orn, Hly, Cth, C-C, Gpr, Php), were identified. The total urinary concentration of AAs in both groups was similar at baseline at approximately $5300 \mathrm{nmol} \mathrm{mL}^{-1}$. The predominant AAs were Gly, His and Php. The main AA fractions were NEAAs which accounted for $43 \%$ of total AAs, EAAs accounted for approximately $27 \%$, and BCAAs-for $5 \%$ of total AAs.

At baseline, the urine profile and the concentrations of individual AAs and total AA did not differ between the experimental groups, whereas noteworthy changes in the urinary excretion of individual AAs were observed after the dietary intervention (Table 3). The urinary excretion of Asn, Lys and Aaa increased significantly $(p<0.05)$ in the Synergy 1 group, and the excretion of Asp and Met decreased $(p<0.05)$ in the placebo group relative to baseline values. Interestingly, the urinary concentration of Glu in both experimental groups decreased by half independently on applied supplement. An analysis of individual AA concentrations in urine after the intervention revealed higher excretion of Ala, Asn, Gln, Phe and Apa in the prebiotic group than in the placebo group. Consequently, urinary excretion of total AAs, 


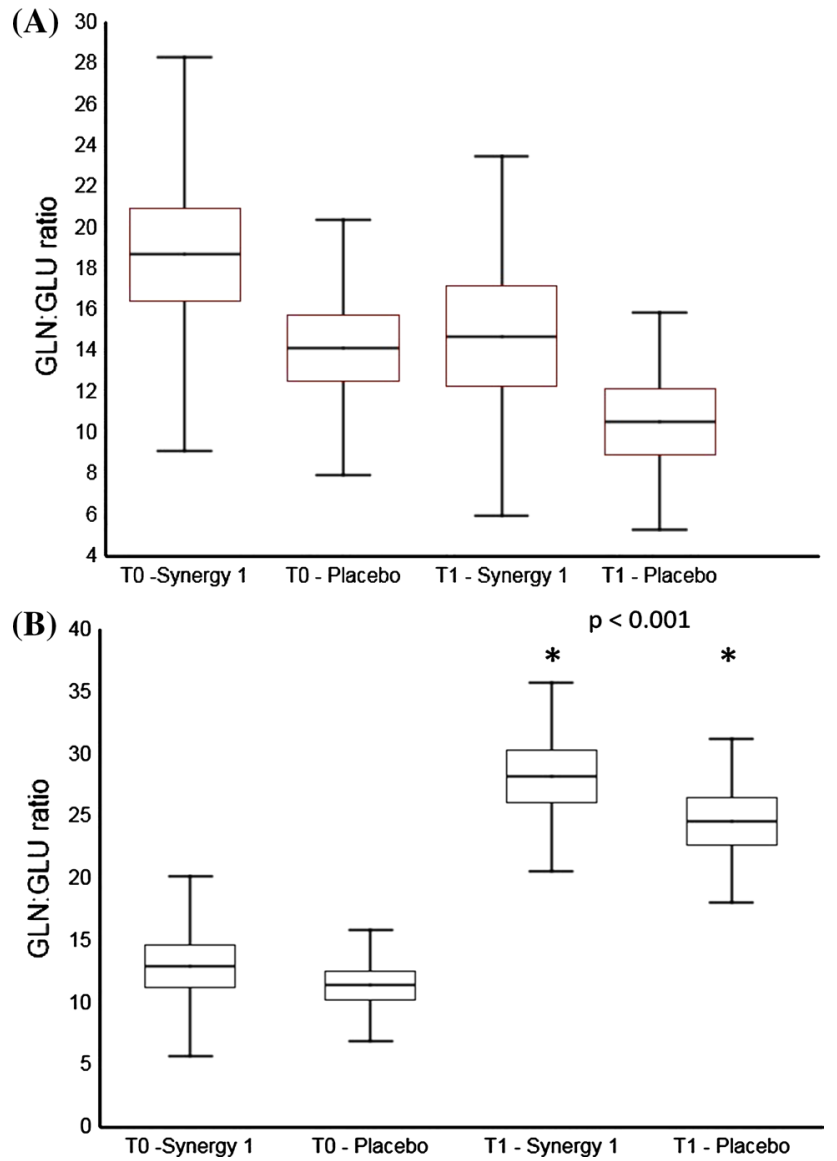

Fig. 1 Box-and-whisker plots of the Gln:Glu ratio in the plasma a and urine $\mathbf{b}$ of children with celiac disease: a comparison of the group supplemented with Synergy 1 and the placebo group at baseline and after 3 months of supplementation; Mann-Whitney test

EAAs and NEAAs increased by more than 14, 25 and 18\%, respectively, in the Synergy 1 group, whereas a decrease in total AA concentrations was observed in the placebo group (Fig. 2b). In the Synergy 1 group, urinary excretion of BCAAs increased by $32 \%$ from baseline and was higher than in the placebo group where only a $4 \%$ increase was noted. The urinary Gln:Glu ratio was calculated for individual patients in each group, and the results are presented in Fig. 1b. The Gln:Glu ratio increased significantly in both groups after the intervention, compared with the baseline value.

\section{Discussion}

The disturbances in the intestinal microbiota, mainly lower number and variety of Bifidobacterium sp. being capable of digesting immunogenic gliadin peptides, have great importance in CD pathophysiology and affect the metabolic profiles in biological fluids of CD patients (Cukrowska et al.
2017). In the present study, we analysed the impact of dietary intervention with prebiotic applied as a supplement of a GFD on the profile and the concentration of AAs in the plasma of children with $\mathrm{CD}$. This is the first ever study to analyse plasma AA levels with the use of the EZ:Faast ${ }^{\mathrm{TM}}$ method; therefore, our results cannot be reliably compared with the findings of other authors. To data, only few studies have investigated the AA profile in the urine or plasma of CD patients. Sevinc et al. (Sevinc et al. 2015) noted a lower levels of $\mathrm{Gln}$ and $\mathrm{C}-\mathrm{C}$ and significantly higher concentrations of Ala, Asn, Glu, Hyp, Ile, Leu, Phe, Pro, Ser, Thr and Val in the plasma of children with CD children than in healthy subjects. The analysis of urinary concentrations of AAs in CD patients showed lower levels of Glu and Gln and higher excretion of Gly compared to healthy controls (Bertini et al. 2009). Children with CD who followed a GFD were characterised by higher concentrations of Lys and Arg and lower Gln levels in urine, compared with healthy controls (Di Cagno et al. 2011). The observed variations in the concentrations of individual AAs between health and CD patients could be attributed to differences in dietary patterns, intestinal malabsorption and inflammatory processes (Sevinc et al. 2015; van Hees et al. 2015)

In general, we detected that after the 3-month dietary intervention, regardless the administered supplement, the total plasma concentration of AAs increased in children with $\mathrm{CD}$. The above result could suggest that a strict GFD alone delivers benefits. The total concentrations of EAAs and NEAAs increased in both groups, which suggest that the continuation of GFD over a period of 3 months induced positive changes in the intestines. The scientific data indicated that the strict adherence to a GFD has beneficial effect on CD patients by reducing symptoms and health care consumption (Norström et al. 2012); therefore, it can be suggested a continuous healing of the intestines in all patients could be related to a good adherence to GFD (Table 1). In the literature, a decrease in the concentrations of the above EAAs has been related to severe intestinal failure, and those EAAs as well as citrulline are regarded as markers of mucosal damage (Fragkos et al. 2015). Interestingly, the plasma concentrations of selected AAs, including Ser, Leu, Thr, Met, Phe, Trp, Aba, Hyp and C-C, increased only in the placebo group. The observed differences in plasma AA levels between groups could be attributed to the specific properties of the administered prebiotic. Prebiotics are substrates which are utilised by gut microbiota during the formation of short-chain fatty acids (SCFAs). Davila et al. (Davila et al. 2013) suggested that AAs could be used by prebiotic-activated gut microbiota to synthesise SCFAs. Gly, Ala, Thr, Glu, Lys and Asp are the precursors of acetate, whereas butyrate is derived from Glu and Lys, and propionate is synthesised from Ala and Thr (Davila et al. 2013). In our previous study, SCFA levels increased in children with 
Fig. 2 Summarized values of total concentrations of all, essential (EAAs), non-essential (NEAAs) and branched-chain (BCAAs) amino acids in plasma $\mathbf{a}$ and urine $\mathbf{b}$ of children with $\mathrm{CD}$ from Synergy 1 and placebo group, before (T0) and after (T1) the intervention
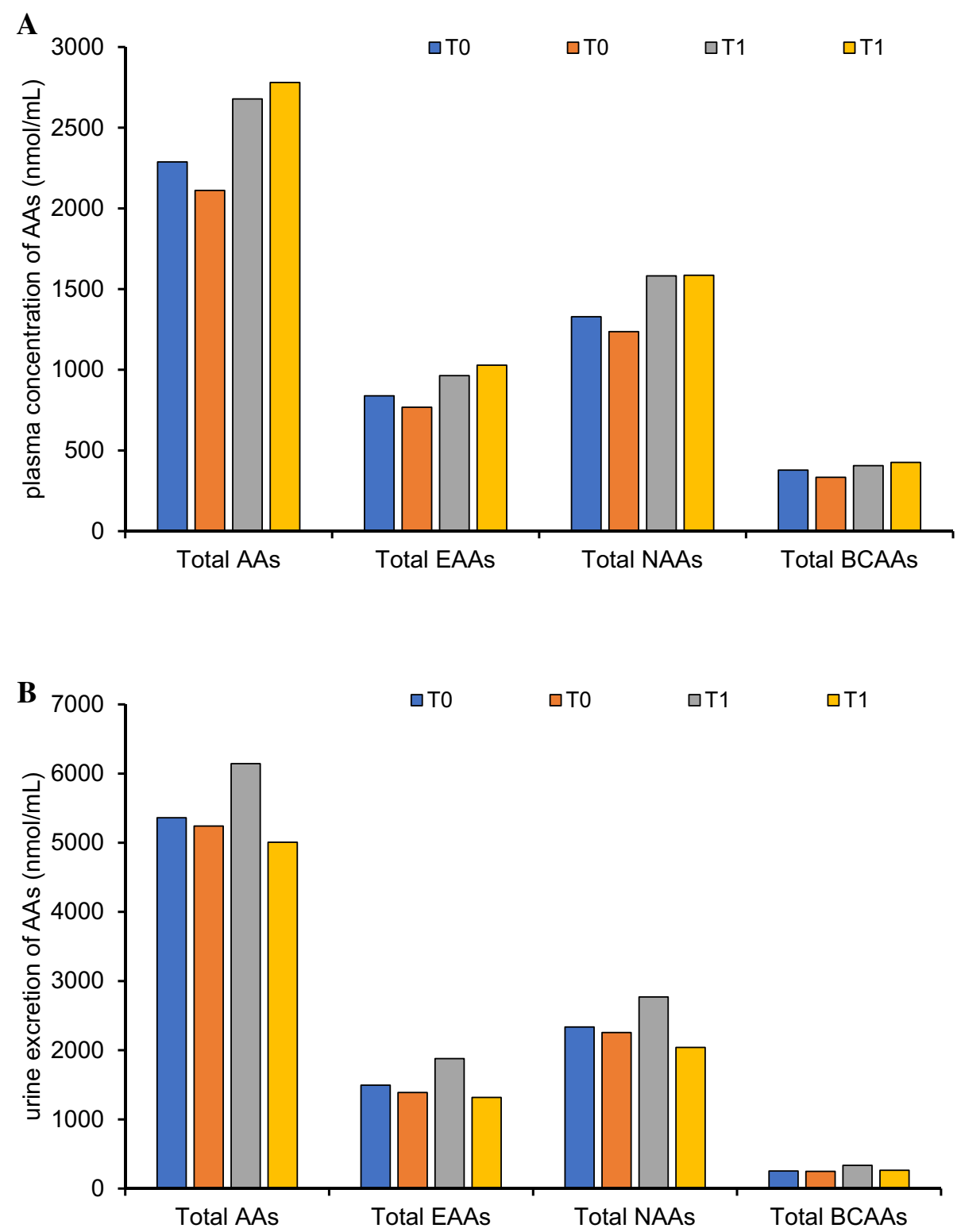

CD after the administration of Synergy 1 (Drabińska et al. 2018). Therefore, lower levels of some individual AAs in the Synergy 1 group compared to the placebo group could suggest that these AAs are transformed in the metabolic pathway and/or are utilised for the production of SCFAs.

In the present study, we noted that the supplementation of GFD with Synergy 1 increased the total AA concentration in urine. Increased urinary excretion of AAs with a simultaneous increase in the content of circulating AAs could be attributed to higher absorption and intensified metabolism of AAs. Therefore, the simultaneous analysis of AAs in urine and plasma seems to be reasonable to avoid misinterpretation. On the other hand, higher urinary excretion of AAs could be associated with the loss of AA caused by metabolic failure, including intestinal permeability and failure in the kidney reabsorption processes (Dunstan et al. 2017). Urinary excretion of AAs decreases in consequence of biochemical and metabolic disorders (Evans et al. 2008) or impaired protein synthesis (Niblett et al. 2007). In a group of autistic patients following a gluten-free and casein-free diet, urinary excretion of AAs increased to a level approximating that noted in healthy controls (Evans et al. 2008). Similar tendency was observed in our study, suggesting positive changes in the metabolism.

Positive changes in Glu concentration were noted after the conducted dietary intervention. In plasma, the concentration of Glu nearly doubled in the Synergy 1 group relative to baseline values, whereas only a $50 \%$ increase was noted in the placebo group. Moreover, urinary excretion of Glu decreased in both groups. Glu is a key factor 


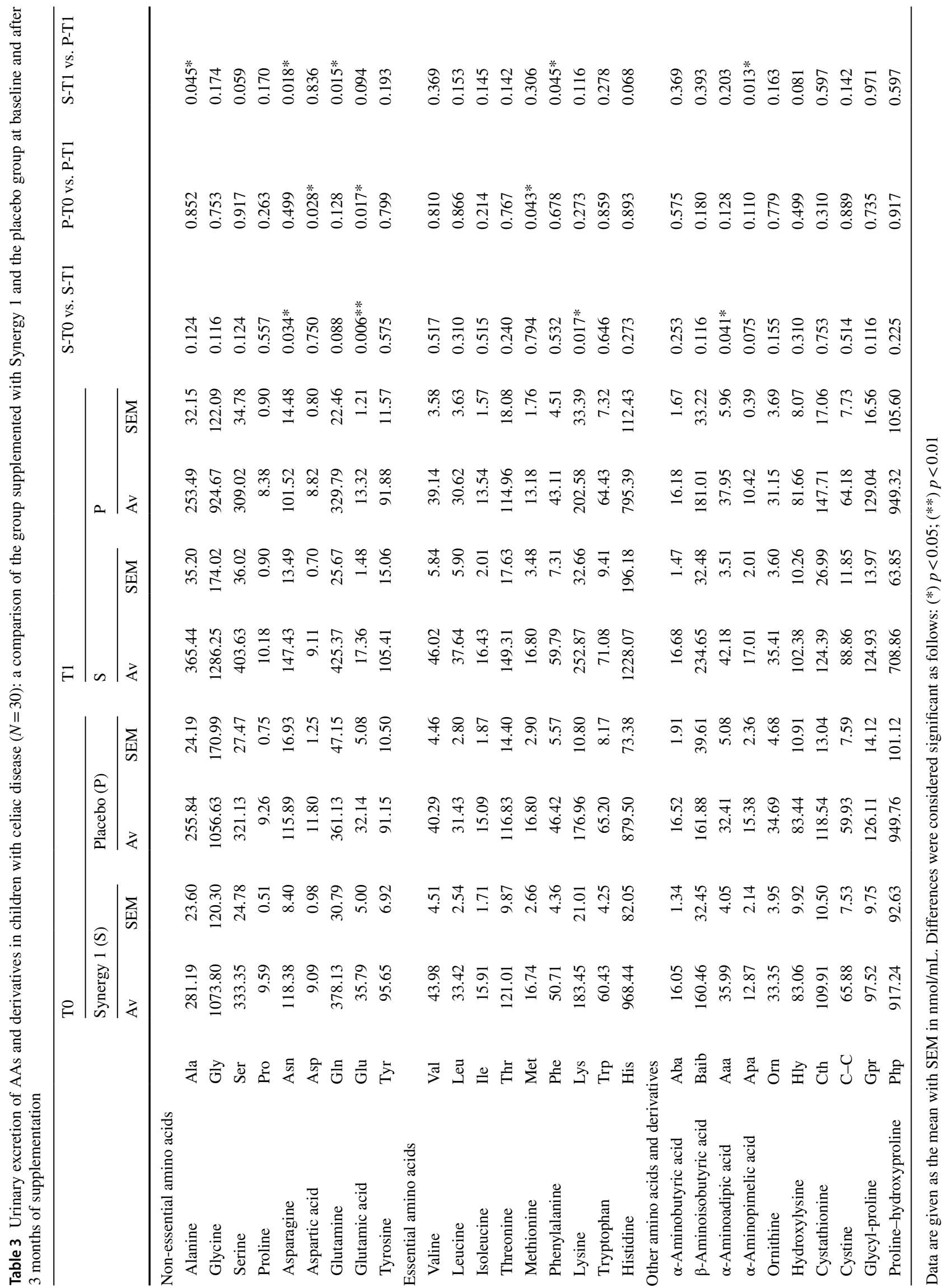


responsible for the maintenance of gut function. Plasma Glu levels are relatively low because this AA is the main substrate for intestinal epithelial cells and a precursor in the synthesis of other AAs (Ala, Aap, Orn, Pro) and mediators (glutathione) (Ruth and Field 2013). There is evidence to indicate that Glu plays an important role in the intestinal barrier function. The supplementation of animal diets with Glu changed intestinal morphology, increased villus height and mucosal thickness in the jejunum ( $\mathrm{Wu}$ et al. 2012). In the Synergy 1 group, the concentration of Gln, a precursor in Glu synthesis, was characterised by a minor increase in plasma samples and a significant increase in urine samples relative to the placebo group. Gln also plays an important role in the gut function, participates in the synthesis of other AAs and protein, influences redox homeostasis and the immune response (Wang et al. 2009; Ruth and Field 2013). Numerous in vitro and in vivo studies have shown that Gln plays a pivotal role in the maintenance of intestinal integrity (DeMarco et al. 2003; Nose et al. 2010). According to Guarner (Guarner 2007), prebiotics contribute to the preservation of gut barrier integrity. Inulin-enriched diets improved intestinal architecture in rats and mice (Kleessen et al. 2003; Strugala et al. 2003; Liu et al. 2016). In most randomised human trials, inulin had no effect on intestinal permeability in patients with burn injuries, critically ill patients or healthy individuals (Jain et al. 2004; Olguin et al. 2005; Ten Bruggencate et al. 2006). Despite the above, Russo et al. observed that inulin modified the concentrations of two intestinal permeability markers, zonulin and glucagonlike peptide 2, and suggested that inulin could be used in the prevention of intestinal disorders (Russo et al. 2012). Further research is needed to explore the ambiguous influence of prebiotics on intestinal barrier function.

The GIn:Glu ratio plays an important role in nitrogen metabolism, brain functions and the gut balance (Hashimoto et al. 2005; Abu Shmais et al. 2012). In the present study, the Gln:Glu ratio was determined in both urine and plasma. Significant changes in Gln:Glu ratio were not observed in plasma, but the analysed parameter increased significantly in urine in both groups after the intervention. These findings could suggest that GFD on its own induces beneficial changes in the intestines. A recent study of patients with chronic intestinal pseudo-obstruction demonstrated a significant decrease $(p<0.001)$ in the urinary Gln:Glu ratio in patients diagnosed with intestinal failure in comparison with healthy controls (Yan et al. 2017).

The present pilot study has certain limitations. The number of participants was low, and the size of the sample and the power of the study could not be determined due to the lack of information from previous studies. However, these limitations are generally associated with pilot studies, and the obtained data will be used to calculate the necessary sample size in future studies. Due to the limited number of participants, a large-scale study should be performed to confirm the results obtained in this pilot study. Last, the present trial was conducted on children whose metabolism differs from that of adults. Therefore, the following study should be performed on older patients to generalise the findings of this research.

\section{Conclusions}

The present randomised placebo-controlled study makes a pioneering attempt to evaluate the influence of a dietary prebiotic on the AA profile of children diagnosed with $\mathrm{CD}$ and following a GFD. Our results indicate that prebioticsupplemented GFD impact the overall AAs metabolism in children with $\mathrm{CD}$, determined in two types of bodily fluids, plasma and urine. In group supplemented with Synergy 1, an increased urinary excretion of AAs with a simultaneous increase in the content of circulating AAs was detected that could be attributed to higher absorption and/or intensified metabolism of AAs, as well as further healing of the intestinal mucosa. The observed changes in Glu concentration suggest that oligofructose-enriched inulin could improve the intestinal condition and permeability. Further research is needed to explore the effect of oligofructose-enriched inulin on intestinal permeability.

Acknowledgements The research was supported by statutory funds of the Department of Chemistry and Biodynamics of Food in the Institute of Animal Reproduction and Food Research PAS. The article is a part of a PhD Thesis of Natalia Drabińska.

Author contributions UKK and ND conceived the study. UKK, ND and EJC were involved in designing of the study. ND collected samples, performed the main part of experiments, analysed the results and drafted the manuscript, EC participated in the chromatographic analysis; ND UKK and EJC contributed to the further writing of the manuscript. All authors reviewed and approved the final version of manuscript.

\section{Compliance with ethical standards}

Conflict of interest As authors of this manuscript, we declare no conflict of interest, either financial or otherwise.

Research involving human participants and/or animals This study had been approved by the Bioethics Committee of the Faculty of Medicine of the University of Warmia and Mazury in Olsztyn, Poland (decision No. 23/2015 of 16 June 2015). The study protocol and all procedures performed in this study involving human participants were done in accordance with the ethical principles of the World Medical Association (WMA) 1964 Declaration of Helsinki and its later amendments or comparable ethical standards. This article does not contain or report any studies with animals performed by any of the authors. The study was registered at www.clinicaltrials.gov as NCT03064997. 
Informed consent Written informed consent was obtained from the parents or legal guardians of all study participants.

Open Access This article is distributed under the terms of the Creative Commons Attribution 4.0 International License (http://creativeco mmons.org/licenses/by/4.0/), which permits unrestricted use, distribution, and reproduction in any medium, provided you give appropriate credit to the original author(s) and the source, provide a link to the Creative Commons license, and indicate if changes were made.

\section{References}

Abu Shmais GA, Al-Ayadhi LY, Al-Dbass AM, El-Ansary AK (2012) Mechanism of nitrogen metabolism-related parameters and enzyme activities in the pathophysiology of autism. J Neurodev Disord 4:1-11. https://doi.org/10.1186/1866-1955-4-4

Addolorato G, Mirijello A, D’Angelo C et al (2008) State and trait anxiety and depression in patients affected by gastrointestinal diseases: psychometric evaluation of 1641 patients referred to an internal medicine outpatient setting. Int J Clin Pract 62:1063-1069. https ://doi.org/10.1111/j.1742-1241.2008.01763.x

Barker JM, Liu E (2008) Celiac disease: pathophysiology, clinical manifestations, and associated autoimmune conditions. Adv Pediatr 55:349-365

Bertini I, Calabró A, De Carli V et al (2009) The metabonomic signature of celiac disease. J Proteome Res 8:170-177. https://doi. org/10.1021/pr800548z

Carta MG, Hardoy MC, Boi MF et al (2002) Association between panic disorder, major depressive disorder and celiac disease: a possible role of thyroid autoimmunity. J Psychosom Res 53:789-793. https ://doi.org/10.1016/S0022-3999(02)00328-8

Cukrowska B, Sowińska A, Bierła JB et al (2017) Intestinal epithelium, intraepithelial lymphocytes and the gut microbiota-key players in the pathogenesis of celiac disease. World J Gastroenterol 23:7505-7518

Davila AM, Blachier F, Gotteland M et al (2013) Re-print of "intestinal luminal nitrogen metabolism: role of the gut microbiota and consequences for the host". Pharmacol Res 69:114-126

DeMarco VG, Li N, Thomas J et al (2003) Glutamine and barrier function in cultured caco-2 epithelial cell monolayers. J Nutr 133:2176-2179

Dereziński P, Klupczynska A, Sawicki W et al (2017) Amino acid profiles of serum and urine in search for prostate cancer biomarkers: a pilot study. Int J Med Sci 14:1-12. https://doi.org/10.7150/ ijms. 15783

Di Cagno R, Rizzello CG, Gagliardi F et al (2009) Different fecal microbiotas and volatile organic compounds in treated and untreated children with celiac disease. Appl Environ Microbiol 75:3963-3971. https://doi.org/10.1128/AEM.02793-08

Di Cagno R, De Angelis M, De Pasquale I et al (2011) Duodenal and faecal microbiota of celiac children: molecular, phenotype and metabolome characterization. BMC Microbiol 11:219. https://doi. org/10.1186/1471-2180-11-219

Drabińska N, Jarocka-Cyrta E, Markiewicz LH, Krupa-Kozak U (2018) The effect of oligofructose-enriched inulin on faecal bacterial counts and microbiota-associated characteristics in celiac disease children following a gluten-free diet: results of a randomized. Placebo Controlled Trial Nutr 10:201. https://doi.org/10.3390/nu100 20201

Dunstan RH, Sparkes DL, Macdonald MM et al (2017) Diverse characteristics of the urinary excretion of amino acids in humans and the use of amino acid supplementation to reduce fatigue and sub-health in adults. Nutr J 16:19. https://doi.org/10.1186/s1293 7-017-0240-y

Evans C, Dunstan RH, Rothkirch T et al (2008) Altered amino acid excretion in children with autism. Nutr Neurosci 11:9-17. https ://doi.org/10.1179/147683008X301360

Fillmann H, Kretzmann NA, San-Miguel B et al (2007) Glutamine inhibits over-expression of pro-inflammatory genes and downregulates the nuclear factor kappaB pathway in an experimental model of colitis in the rat. Toxicology 236:217-226. https://doi. org/10.1016/j.tox.2007.04.012

Fragkos KC, Samaan M, Morgan S et al (2015) PTH-200 amino acid profiles in patient with intestinal failure: preliminary data on biochemical insights. Gut 64:A497 LP-A498 LP

Guarner F (2007) Studies with inulin-type fructans on intestinal infections, permeability, and inflammation. J Nutr 137:2568S-2571S 137/11/2568S

Hashimoto K, Engberg G, Shimizu E et al (2005) Elevated glutamine/glutamate ratio in cerebrospinal fluid of first episode and drug naive schizophrenic patients. BMC Psychiatry 5:6. https://doi.org/10.1186/1471-244X-5-6

Hernanz A, Polanco I (1991) Plasma precursor amino acids of central nervous system monoamines in children with coeliac disease. Gut 32:1478-1481. https://doi.org/10.1136/gut.32.12.1478

Hugh Dunstan R, Sparkes DL, Macdonald MM et al (2011) Altered amino acid homeostasis and the development of fatigue by breast cancer radiotherapy patients: a pilot study. Clin Biochem 44:208-215. https://doi.org/10.1016/j.clinbiochem.2010.10.002

Husby S, Koletzko IR, Korponay-Szabó ML et al (2012) European society for pediatric gastroenterology, hepatology, and nutrition guidelines for the diagnosis of coeliac disease. J Pediatr Gastroenterol Nutr 54:136-160. https://doi.org/10.1097/MPG.0b013 e31821a23d0

Ilus T, Kaukinen K, Virta LJ et al (2014) Incidence of malignancies in diagnosed celiac patients: a population-based estimate. Am J Gastroenterol 109:1471-1477. https://doi.org/10.1038/ ajg.2014.194

Jain PK, McNaught CE, Anderson ADG et al (2004) Influence of synbiotic containing lactobacillus acidophilus La5, bifidobacterium lactis $\mathrm{Bb} 12$, streptococcus thermophilus, lactobacillus bulgaricus and oligofructose on gut barrier function and sepsis in critically ill patients: a randomised controlled tr. Clin Nutr 23:467-475. https ://doi.org/10.1016/j.clnu.2003.12.002

Kleessen B, Hartmann L, Blaut M (2003) Fructans in the diet cause alterations of intestinal mucosal architecture, released mucins and mucosa-associated bifidobacteria in gnotobiotic rats. Br J Nutr 89:597. https://doi.org/10.1079/BJN2002827

Krupa-Kozak U, Swiątecka D, Bączek N, Brzóska MM (2016) Inulin and fructooligosaccharide affect in vitro calcium uptake and absorption from calcium-enriched gluten-free bread. Food Funct 7:1950-1958. https://doi.org/10.1039/c6fo00140h

Krupa-Kozak U, Drabińska N, Jarocka-Cyrta E (2017) The effect of oligofructose-enriched inulin supplementation on gut microbiota, nutritional status and gastrointestinal symptoms in paediatric coeliac disease patients on a gluten-free diet: study protocol for a pilot randomized controlled trial. Nutr J 16:47. https://doi.org/10.1186/ s12937-017-0268-z

Liu T-W, Cephas KD, Holscher HD et al (2016) Nondigestible fructans alter gastrointestinal barrier function, gene expression, histomorphology, and the microbiota profiles of diet-induced obese C57BL/6 J Mice. J Nutr 146:949-956. https://doi.org/10.3945/ jn.115.227504

Niblett SH, King KE, Dunstan RH et al (2007) Hematologic and urinary excretion anomalies in patients with chronic fatigue syndrome. Exp Biol Med (Maywood) 232:1041-1049. https://doi. org/10.3181/0702-RM-44 
Norström F, Sandström O, Lindholm L, Ivarsson A (2012) A glutenfree diet effectively reduces symptoms and health care consumption in a Swedish celiac disease population. BMC Gastroenterol 12:125. https://doi.org/10.1186/1471-230X-12-125

Nose K, Yang H, Sun X et al (2010) Glutamine prevents total parenteral nutrition-associated changes to intraepithelial lymphocyte phenotype and function: a potential mechanism for the preservation of epithelial barrier function. J Interferon Cytokine Res 30:67-80. https://doi.org/10.1089/jir.2009.0046

Olguin F, Araya M, Hirsch S et al (2005) Prebiotic ingestion does not improve gastrointestinal barrier function in burn patients. Burns 31:482-488. https://doi.org/10.1016/j.burns.2004.11.017

Ren W, Yin J, Wu M et al (2014) Serum amino acids profile and the beneficial effects of L-arginine or L-glutamine supplementation in dextran sulfate sodium colitis. PLoS One 9:e88335. https://doi. org/10.1371/journal.pone.0088335

Roma E, Roubani A, Kolia E et al (2010) Dietary compliance and life style of children with coeliac disease. J Hum Nutr Diet 23:176182. https://doi.org/10.1111/j.1365-277X.2009.01036.x

Russo F, Linsalata M, Clemente C et al (2012) Inulin-enriched pasta improves intestinal permeability and modifies the circulating levels of zonulin and glucagon-like peptide 2 in healthy young volunteers. Nutr Res 32:940-946. https://doi.org/10.1016/j.nutre s.2012.09.010

Ruth MR, Field CJ (2013) The immune modifying effects of amino acids on gut-associated lymphoid tissue. J Anim Sci Biotechnol $4: 27$

Sevinc E, Akar HH, Sevinc N et al (2015) Amino acid levels in children with celiac disease. Nutr Hosp 32:139-143. https://doi. org/10.3305/nh.2015.32.1.9066
Shoaib M, Shehzad A, Omar M et al (2016) Inulin: properties, health benefits and food applications. Carbohydr Polym 147:444-454

Strugala V, Allen A, Dettmar PW, Pearson JP (2003) Colonic mucin: methods of measuring mucus thickness. Proc Nutr Soc 62:237243. https://doi.org/10.1079/PNS2002205

Ten Bruggencate SJM, Bovee-Oudenhoven IMJ, Lettink-Wissink MLG et al (2006) Dietary fructooligosaccharides affect intestinal barrier function in healthy men. J Nutr 136:70-74

van Hees NJM, Giltay EJ, Tielemans SMAJ et al (2015) Essential amino acids in the gluten-free diet and serum in relation to depression in patients with celiac disease. PLoS One 10:e0122619. https ://doi.org/10.1371/journal.pone.0122619

Wang WW, Qiao SY, Li DF (2009) Amino acids and gut function. Amino Acids 37:105-110

Wu G, Fang Y-Z, Yang S et al (2004) Glutathione metabolism and its implications for health. J Nutr 134:489-492 (14988435)

Wu X, Zhang Y, Liu Z et al (2012) Effects of oral supplementation with glutamate or combination of glutamate and $\mathrm{N}$-carbamylglutamate on intestinal mucosa morphology and epithelium cell proliferation in weanling piglets. J Anim Sci 90:337-339. https://doi. org/10.2527/jas.53752

Yan JK, Zhou KJ, Huang JH et al (2017) Urinary glutamine/glutamate ratio as a potential biomarker of pediatric chronic intestinal pseudo-obstruction. Orphanet J, Rare Dis, p 12

Zhang H, Hu CAA, Kovacs-Nolan J, Mine Y (2015) Bioactive dietary peptides and amino acids in inflammatory bowel disease. Amino Acids 47:2127-2141. https://doi.org/10.1007/s00726-014-1886-9 